\title{
BRCA testing in a genomic diagnostics referral center during the COVID-19 pandemic
}

\author{
Angelo Minucci ${ }^{1}\left[\right.$ Giovanni Scambia $^{2,3} \cdot$ Concetta Santonocito $^{1,3} \cdot$ Paola Concolino ${ }^{1} \cdot$ Andrea Urbani $^{1,3}$
}

Received: 8 April 2020 / Accepted: 28 April 2020 / Published online: 9 May 2020

(c) Springer Nature B.V. 2020

\begin{abstract}
The first person-to-person transmission of the 2019-novel coronavirus in Italy on 21 February 2020 led to an infection chain that represents one of the largest known COVID-19 outbreaks outside Asia. Hospitals have been forced to reorganized their units in response to prepare for an unforeseen healthcare emergency. In this context, our laboratory (Molecular and Genomic Diagnostics Unit, Fondazione Policlinico Universitario Agostino Gemelli IRCCS) re-modulated its priorities by temporarily interrupting most of the molecular tests guaranteeing only those considered "urgent" and not postponable. In particular, this paper details changes regarding the execution of germline $B R C A(g B R C A)$ testing in our laboratory. A substantial reduction in $g B R C A$ testing (about 60\%) compared to the first 2 months of the current year was registered, but the requests have not been reset. The requesting physicians were mainly gynaecologists and oncologists. These evidences further emphasize the new era of $g B R C A$ testing in the management of cancer patients and confirms definitively the integration of $g B R C A$ testing/ Next Generation Sequencing (NGS) into clinical oncology. Finally, a re-organization of $g B R C A$ testing in our Unit, mainly related to delayed and reduced arrival of tests was necessary, ensuring, however, a high-quality standard and reliability, mandatory for $g B R C A$ testing in a clinical setting.
\end{abstract}

Keywords BRCA genes · Germline BRCA testing · COVID-19 pandemic in Italy · Coronavirus disease 2019

$\begin{array}{ll}\text { Abbreviations } \\ \text { CNA } & \text { Copy number alteration } \\ \text { COVID-19 } & \text { Coronavirus disease-2019 } \\ \text { gBRCA } & \text { Germline } B R C A \\ \text { HPC } & \text { Hereditary prostate cancer } \\ \text { NGS } & \text { Next Generation Sequencing } \\ \text { PA } & \text { Pancreatic adenocarcinoma } \\ \text { PARP-1 } & \text { Poly-(ADP-ribose) polymerase } \\ \text { PC } & \text { Prostate cancer } \\ \text { PV } & \text { Pathogenic variant }\end{array}$

Angelo Minucci

angelo.minucci@policlinicogemelli.it; angelo.minucci@virgilio.it

$\triangle$ Andrea Urbani andrea.urbani@policlinicogemelli.it

1 Molecular and Genomic Diagnostics Unit, Fondazione Policlinico Universitario Agostino Gemelli IRCCS, Rome, Italy

2 Division of Oncological Gynecology, Department of Women's and Children's Health, Fondazione Policlinico Universitario Agostino Gemelli IRCCS, Rome, Italy

3 Catholic University of the Sacred Heart, Rome, Italy

\section{Introduction}

The entire world is facing a devastating crisis in the growing pandemic associated with the coronavirus diseaseCOVID-19-and Italy is paying dearly for this pandemic both in terms of public health and economics. Since Feb 21, 2020, when the first Italian COVID-19 patient was confirmed, the National Healthcare Service, based on the Beveridge model offering universal and equal health care to all citizens, has faced increasing pressure, with 110,574 total cases of COVID-19 and 13,155 deaths as of April 1, 2020 [1]. On March 8, 2020, the Italian Government implemented extraordinary measures to limit social contacts with the attempt to minimize the likelihood that healthy people come into contact with infected people.

In this special situation, hospitals were forced to reorganize their units to the ongoing emergency.

In this context, physicians, geneticists, molecular biologists and pathologists were concerned about the management of cancer patients. In particular, our laboratory (Molecular and Genomics Diagnostics Unit, Fondazione Policlinico Universitario Agostino Gemelli, IRCCS) remodulated its priorities by temporarily interrupting most 
of the molecular tests and guaranteeing only those considered "urgent" and not postponable.

Herein, we report changes regarding the execution of the germline BRCA ( $g B R C A$ ) testing, during the COVID19 outbreak in March 2020, in a referral center for the gBRCA testing [2-5].

$B R C A$ genes are more involved in hereditary breast and ovarian cancer syndrome, accounting for $30 \%$ to $70 \%$ of hereditary breast cancer and about $90 \%$ of hereditary ovarian cancer families [6]. In addition, BRCA genes increase the risk for pancreatic and prostate cancers (PCs). In fact, approximately $3 \%$ to $10 \%$ of patients with pancreatic adenocarcinoma (PA) have a family history of pancreatic cancer and approximately $10 \%$ to $20 \%$ of PAs are due to a genetic cause [7, 8]. Additionally, PC has some of the highest heritability of any cancer; Scandinavian studies estimate that up to $57 \%$ of the individual variation in risk is due to genetic factors $[9,10]$. However, although the involvement of high-risk genes explains only a small proportion of hereditary prostate cancer (HPC) families, PVs in BRCA2 have been found in only $1.2 \%$ to $3.2 \%$ of patients with PC in most studies, whereas BRCAl PVs are found in even smaller percentages [11-13]. Nonetheless, these genes (particularly BRCA2) remain the most common known causes of HPC.

Considering the above mentioned evidence, we emphasize that in case $g B R C A$ testing is performed only for this purpose, it would be considered a molecular/genetic test, with only important implications of cancer risk-assessment and prevention for relatives of patients with $B R C A$ PVs, which would in consequence not be considered an urgent test, therefore, postponable.

However, cancer patients with test positive for $B R C A$ PVs show a greater benefit from the treatment with poly ADP ribose polymerase inhibitors (PARPi) compared with wild-type patients. In fact, over the past decade, multiple clinical studies have shown that patients harbouring $g B R C A$ PVs are sensitive to PARPi and platinum-based chemotherapy and are associated with improved overall survival. As a result, $g B R C A$ testing has become routine in the clinical management of ovarian and breast cancer patients $[14,15]$. Additionally, more recently, PARPi is playing an increasingly important role in the care of patients with PA and PC; nowadays $g B R C A$ testing is recommended for all patients with pancreatic or metastatic PC as well as patients with high Gleason grade PC $[16,17]$.

In this scenario, $g B R C A$ testing assumes an important role as mandatory molecular test to address patients towards targeted therapies. It has become an urgent molecular test especially when patients are faced with therapeutic crossroads, test results can change their life.

In light of this evidence, our Unit performed about 1000 gBRCA tests in 2019 (Fig. 1) and geneticists, oncologists and gynaecologists, belonging to our institution, were the mainly requesting $g B R C A$ testing. As shown in Fig. 1, a steady increase of tests according with the last acquisitions of the new therapeutic opportunity for PA and PC patients has been registered.

Since $g B R C A$ testing is nowadays performed by Next Generation Sequencing (NGS) [18] our Unit has never had any particular programming issues related to availability of samples to program the sequencing run. We were always able to organize sequencing run using any available Illumina sequencing flow cell format (NGS platform used in our Unit).

With the implementation of the behavioural restrictions by the Italian Government (March 2020) during the COVID19 pandemic, a substantial reduction in $g B R C A$ testing (about 60\%) compared to the first 2 months of the current year was registered, but the requests have not been reset. In detail, 41 patients underwent the test. The requesting physicians were gynecologists, oncologists and geneticists
Fig. 1 Germline $B R C A$ tests performed during the year 2019 and in the first 3 months of the year 2020. In March 2020, in full pandemic there was a substantial reduction in the $g B R C A$ testing (about 60\%) compared to the first 2 months of the current year. In the figure, dates of the first case positive for COVID-19 and starting of the restrictive measures in Italy are reported

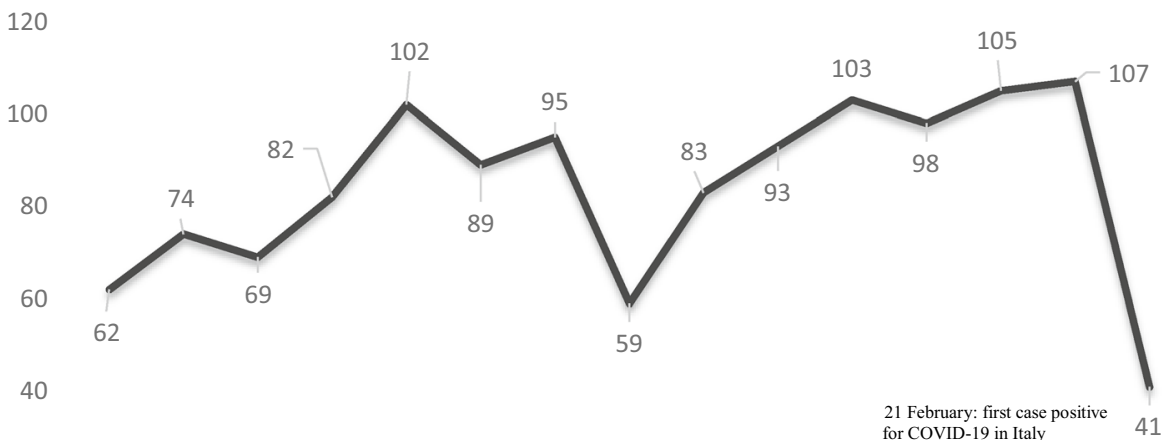

20

11 March: starting of the restrictive measures

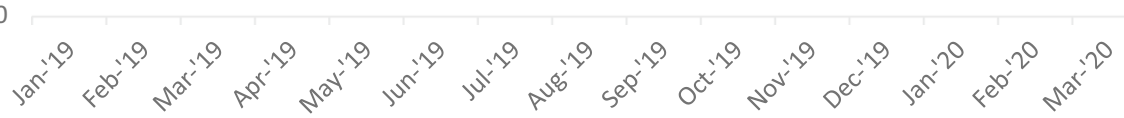


Fig. 2 Clinicians who requested the $g B R C A$ testing during Mach 2020. Most tests performed (80\%) had the aim of directing patients towards personalized therapies, irrespective of personal and/or family history of cancers

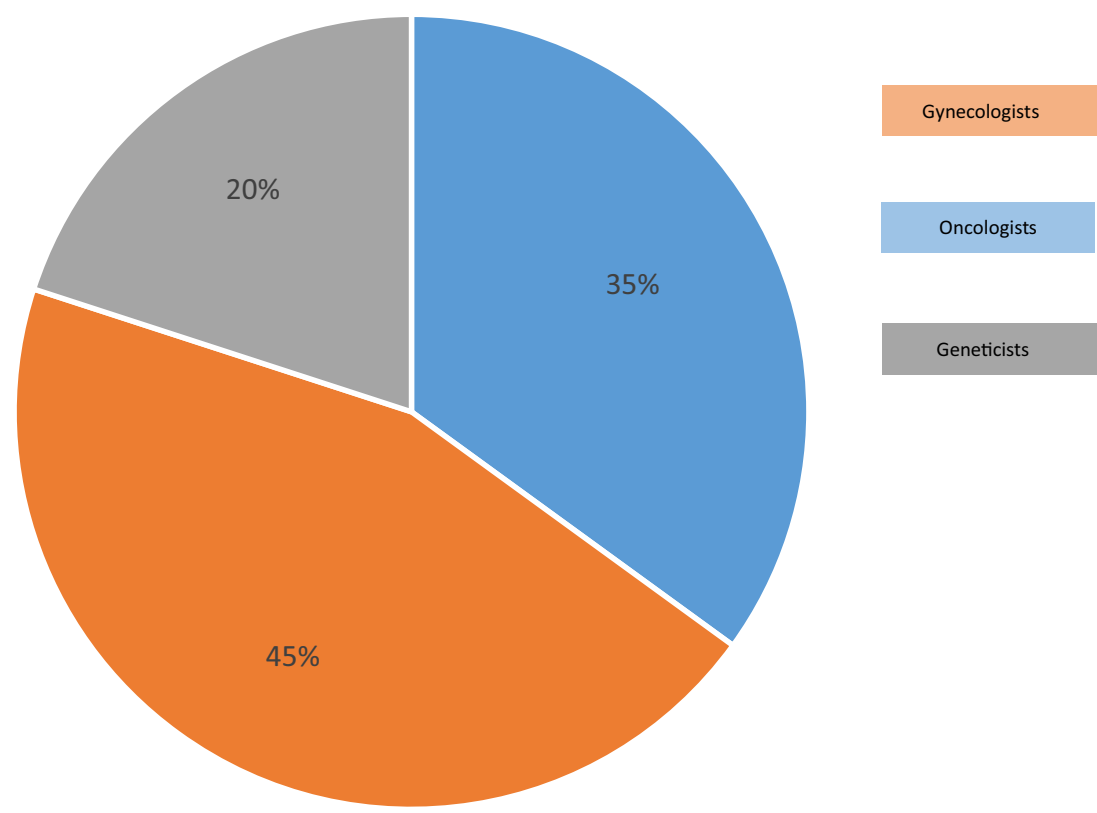

(Fig. 2). Most tests performed (80\%) had the aim of directing patients towards personalized therapies, irrespective of personal and/or family history of cancers.

These evidences further emphasize the new era of $g B R C A$ testing in the management of cancer patients and confirm definitively the integration of $g B R C A$ testing/NGS into clinical oncology. We underline that the use of predictive tests in the forthcoming years for many other molecular markers will most likely increment.

\section{Conclusions}

Even in the presence of a serious ongoing emergency, such as the COVID-19 pandemic, it is evident that cancer patients must continue to take advantage of $g B R C A$ testing to target therapy. This involves a great effort of all actors involved: to begin with clinicians followed by molecular biologists who must review and reorganize $g B R C A$ testing, which is mainly related to delayed and reduced arrival of tests. In our Unit, this has led to use the smallest Illumina sequencing flow cell format due to fewer samples and to programming NGS in a shorter time. At the same time, however, great attention has been paid to reach the right compromise between NGS costs and turnaround time for testing, guaranteeing, however, a high quality standard and reliability, mandatory for $g B R C A$ testing in a clinical setting. In fact, identification of $B R C A$ single nucleotide variants (SNVs) indels and copy number alteration (CNA) (where a minimum of samples is need to have reliable bioinformatics prediction [19]) was obtained.
Finally, we underline that this becomes achievable only when the diagnostics Unit is composed of highly qualified and highly experienced staff.

Acknowledgements We would like to thank Franziska M. Lohmeyer for critically reviewing and editing our manuscript. In addition, we would like to thank Maria De Bonis, Elisa De Paolis, Giorgia Mazzuccato, Roberta Rizza, Maria Elisabetta Onori and Laura Foca for their work dedication even in this particular moment we are experiencing.

\section{Compliance with ethical standards}

Conflict of interest The authors declared no potential conflicts of interest.

Ethical approval This study complied with the Ethical Principles for Medical Research Involving Human Subjects according to the World Medical Association Declaration of Helsinki and was certified by the Committee of the Applicable Institution of the Fondazione Policlinico Universitario Agostino Gemelli IRCCS, Rome.

Informed consent Informed consent was taken from all patients who underwent the $g B R C A$ testing.

\section{References}

1. Ministry of Health of Italy. COVID-19 situazione in Italia. March 19, 2020. http://www.salute.gov.it/portale/nuovocoronavirus/dettaglioContenutiNuovoCoronavirus.jsp?lingua $=\mathrm{italiano \& id}=5351$ \&area=nuovoCoronavirus\& menu=vuoto, accessed Mar 20, 2020

2. Minucci A, Scambia G, Santonocito C et al (2015) Clinical impact on ovarian cancer patients of massive parallel sequencing for BRCA mutation detection: the experience at Gemelli hospital and a literature review. Expert Rev Mol Diagn 15:1383-1403. https:// doi.org/10.1586/14737159.2015.1081059 
3. Minucci A, De Paolis E, Concolino P et al (2017) Competitive PCR-high resolution melting analysis (C-PCR-HRMA) for large genomic rearrangements (LGRs) detection: a new approach to assess quantitative status of BRCA1 gene in a reference laboratory. Clin Chim Acta 470:83-92. https://doi.org/10.1016/j. cca.2017.04.026

4. Santonocito C, Scapaticci M, Guarino D et al (2017) Identification of twenty-nine novel germline unclassified variants of BRCA1 and BRCA2 genes in 1400 Italian individuals. Breast 36:74-78. https ://doi.org/10.1016/j.breast.2017.09.007

5. Concolino P, Rizza R, Mignone F et al (2018) A comprehensive BRCA1/2 NGS pipeline for an immediate Copy Number Variation $(\mathrm{CNV})$ detection in breast and ovarian cancer molecular diagnosis. Clin Chim Acta 480:173-179. https://doi.org/10.1016/j. cca.2018.02.012

6. Gori S, Barberis M, Bella MA et al (2019) Recommendations for the implementation of BRCA testing in ovarian cancer patients and their relatives. Crit Rev Oncol Hematol 140:67-72. https:// doi.org/10.1016/j.critrevonc.2019.05.012

7. Bartsch DK, Gress TM, Langer P (2012) Familial pancreatic cancer: current knowledge. Nat Rev Gastroenterol Hepatol 9:445453. https://doi.org/10.1038/nrgastro.2012.111

8. Petersen GM (2016) Familial pancreatic cancer. Semin Oncol 43:548-553. https://doi.org/10.1053/j.seminoncol.2016.09.002

9. Mucci LA, Hjelmborg JB, Harris JR et al (2016) Nordic Twin Study of Cancer (NorTwinCan) collaboration. Familial risk and heritability of cancer among twins in Nordic countries. JAMA 315:68-76. https://doi.org/10.1001/jama.2015.17703

10. Hjelmborg JB, Scheike T, Holst K et al (2014) The heritability of prostate cancer in the Nordic Twin Study of Cancer. Epidemiol Biomarkers Prev 23:2303-2310. https://doi.org/10.1158/10559965.EPI-13-0568

11. Gallagher DJ, Gaudet MM, Pal P et al (2010) Germline BRCA mutations denote a clinicopathologic subset of prostate cancer. Clin Cancer Res 16:2115-2121. https://doi.org/10.1158/10780432.CCR-09-2871

12. Kote-Jarai Z, Leongamornlert D, Saunders E et al (2011) UKGPCS collaborators. BRCA2 is a moderate penetrance gene contributing to young-onset prostate cancer: implications for genetic testing in prostate cancer patients. Br J Cancer 105:1230 1234. https://doi.org/10.1038/bjc.2011.383

13. Giusti RM, Rutter JL, Duray PH et al (2003) A twofold increase in BRCA mutation related prostate cancer among Ashkenazi Israelis is not associated with distinctive histopathology. J Med Genet 40:787-792. https://doi.org/10.1136/jmg.40.10.787

14. Ledermann J, Harter P, Gourley C et al (2014) Olaparib maintenance therapy in patients with platinum-sensitive relapsed serous ovarian cancer: a preplanned retrospective analysis of outcomes by BRCA status in a randomised phase 2 trial. Lancet Oncol 15:852-861. https://doi.org/10.1016/S1470-2045(14)70228-1

15. Zimmer AS, Gillard M, Lipkowitz S (2018) Update on PARP inhibitors in breast cancer. Curr Treat Options Oncol 19:21. https ://doi.org/10.1007/s11864-018-0540-2

16. Mateo J, Carreira S, Sandhu S (2015) DNA-repair defects and Olaparib in metastatic prostate cancer. N Engl J Med 373:16971708. https://doi.org/10.1056/NEJMoa1506859

17. Golan T, Hammel P, Reni M et al (2019) Olaparib as maintenance treatment following first-line platinum-based chemotherapy in patients with a germline BRCA mutation and metastatic pancreatic cancer: phase III POLO trial. Ann Oncol 30(Suppl 4):iv152. https://doi.org/10.1093/annonc/mdz183

18. Wallace AJ (2016) New challenges for BRCA testing: a view from the diagnostic laboratory. Eur J Hum Genet Suppl 1:S10-S18. https://doi.org/10.1038/ejhg.2016.94

19. Minucci A, Mazzuccato G, Marchetti C et al (2020) Detecting germline rearrangements of BRCA1 by next generation tumor sequencing. Mol Biol (in press)

Publisher's Note Springer Nature remains neutral with regard to jurisdictional claims in published maps and institutional affiliations. 